\title{
The use of antibiotic therapy as an adjunct treatment of bone and joint infections
}

$\mathrm{T}$ HE INFECTIOUS DISEASES AND IMMUNIZATION COMMITTEE of the Canadian Paediatric Society has prepared a thoughtful position paper addressing the role of oral antibiotics in the 'consolidation phase' of therapy of bone and joint infections in otherwise healthy children (page 10-12). Although the committee has restricted the application of its guidelines to children older than three months of age, I believe that the same therapeutic principles are applicable to adults with septic arthritis and osteomyelitis.

Although I know of no data that directly compare the outcome of bone and joint infections treated exclusively with parenteral antibiotics with the outcome of infections treated with sequential intravenous-oral therapy, there is no inherent reason to think that one approach would be superior, provided that a bioequivalent dose of drug is given by both routes. In general, as outlined by the authors of the position paper, the dosage of oral antibiotic selected should be higher than that used for the usual minor infection treated in ambulatory patients. The use of a higher dose is designed to assure the desirable bioequivalency. Also, as emphasized by the authors, it is important to demonstrate that the selected dose results in adequate blood concentrations. This adequacy can be determined by directly measuring drug concentrations or by measuring serum inhibitory and bactericidal concentrations. The advantage of using serum inhibitory and bactericidal concentrations is that they can be performed easily by most laboratories, and their results not only reflect the amount of antibiotic that the patient is absorbing but also the effect of nonspecific immune factors present in the patient's serum that are active against the infecting pathogen.

In general, I agree with the statements made by the Committee, but I would tend to be more liberal in determining which patients could be eligible for oral antibiotic therapy. The committee has noted that patients with more than seven days of signs and symptoms before therapy are less likely to have successful outcome with oral therapy, implying that it should not be used in these subjects. I agree that a delay in therapy may compromise the likelihood of a favourable outcome, but I do not think delayed therapy precludes the ultimate use of oral antibiotics. Indeed, the first use of oral antibiotics was in patients with chronic osteomyelitis. This also is the group that stands to gain the most from oral therapy as such patients often need to be treated for months or even years.

The committee also requires the isolation of a pathogen before considering the use of oral therapy. Committee members estimate that with bone and/or joint aspiration a pathogen can be isolated $85 \%$ of the time. I think that the absence of an isolate is much more frequent, either because the appropriate culture was not obtained, the culture was falsely negative, or the patient was not infected. Irrespective of the correct explanation for negative cultures, if the decision is made to continue antibiotic therapy, I would not deny these 'culture-negative' patients the advantages of switching from the intravenous to the oral route. In the absence of a positive culture, the antibiotic selection for these patients should be designed to provide the same spectrum of coverage as their initially selected empirical regimen. For cases of osteomyelitis, coverage for Staphylococcus aureus is most critical, and for cases of septic arthritis coverage for $S$ aureus, streptococci and Neisseriae species needs to be incorporated into the empirical selection. Cases of bone and joint infection caused by Haemophilus influenzae type b probably no longer need to be considered even in young children if they have been vaccinated against $H$ influenzae type $b$.

When selecting the most appropriate oral antibiotic, 
it not only is necessary to consider the in vitro antimicrobial activity of the agent, but it also is important to consider the drug's side effect profile, dosing schedule, cost, formulation and palatability. Patients are more likely to comply with a well tolerated, infrequently administered, affordable drug than a poorly tolerated, expensive agent that needs to be taken throughout the day and night. Formulation and taste are most relevant to the treatment of infections in children. Data are available that rank the palatability of different agents, but the final determination of acceptance in any given patient probably rests with their taste buds. It is the physician's responsibility to determine if the parents are able to coax their child into taking the prescribed medication and to change therapy if the drug is 'rejected'.

I agree with the committee that the close monitoring of patients during their oral phase of therapy is critical to success. Such monitoring should be accomplished at weekly clinic visits. During these visits the interim history should be elicited, the affected bone/joint examined and an inhibitory/bactericidal titre obtained. If a patient has never had bacteria isolated, these titres could be performed with the use of a laboratory strain of $S$ aureus. This titre would serve as a surrogate measure of compliance and would provide reassurance that absorption of the antibiotic remains adequate. I am uncertain as to the value of determining serial erythrocyte sedimentation rates as they are so nonspecific. I know of no data demonstrating this test as "an excellent predictor of successful outcome" as stated by the committee.

The Infectious Diseases and Immunization Committee of the Canadian Paediatric Society should be commended on their effort to simplify the therapy of bone and joint infections. Their statement is timely considering the major efforts that are being made throughout North America to minimize the duration of hospitalization in an attempt to reduce the costs of health care. I believe that the management of bone and joint infections in children and adults can be made less expensive through the prudent use of oral therapy. However, we must continue to recognize that these are severe infections with the potential for substantial morbidity if appropriate monitoring is not accomplished.

Charles G Prober MD

Professor of Pediatrics, Medicine, Microbiology and Immunology, Associate Chairman. Department of Pediatrics, Stanford University School of Medicine, Department of Pediatrics, Division of Infectious Diseases Stanford University Medical Center, Stanford, California 


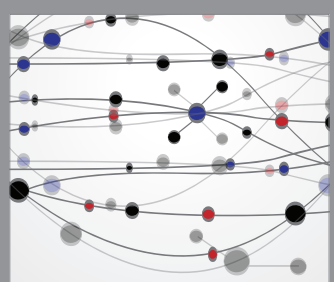

The Scientific World Journal
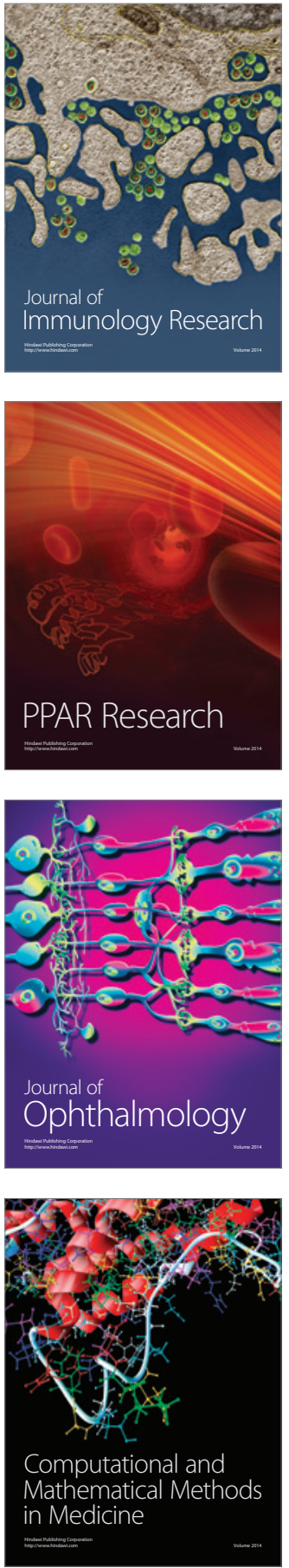

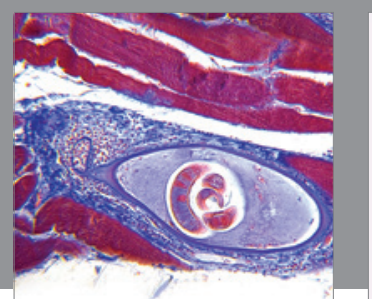

Gastroenterology Research and Practice

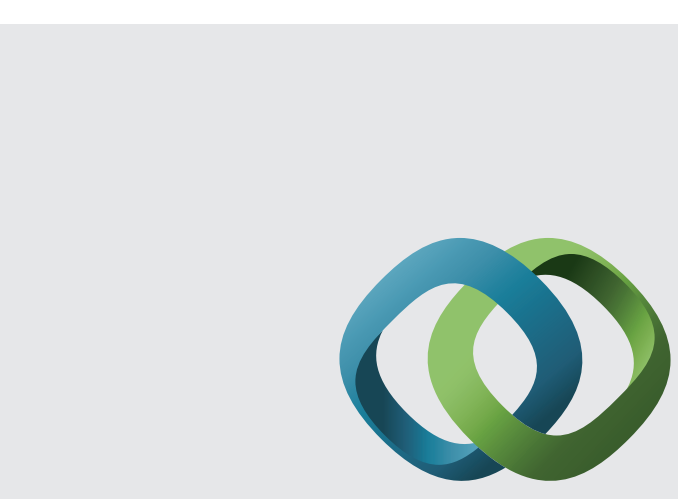

\section{Hindawi}

Submit your manuscripts at

http://www.hindawi.com
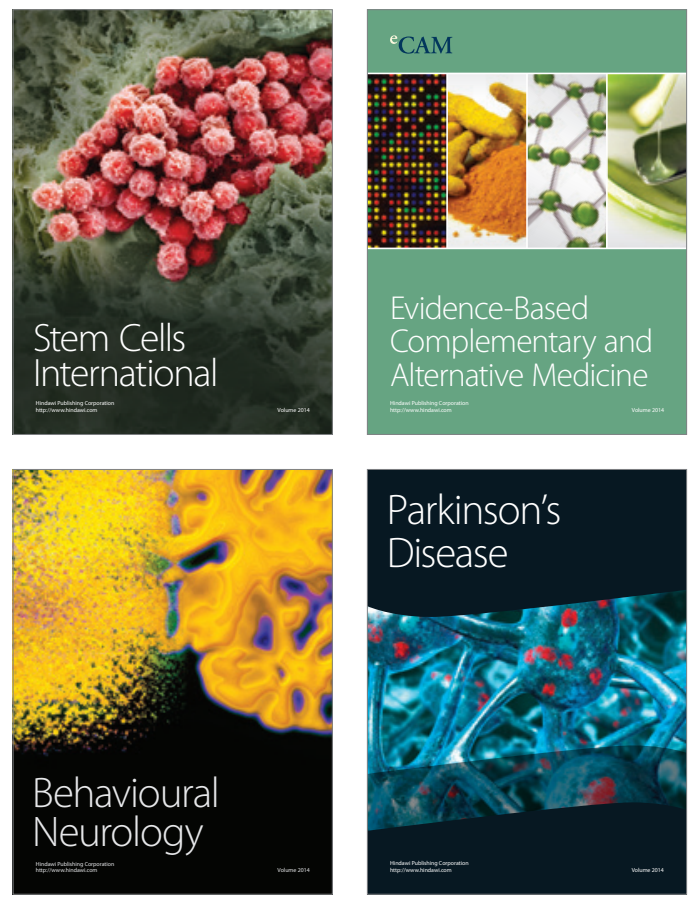
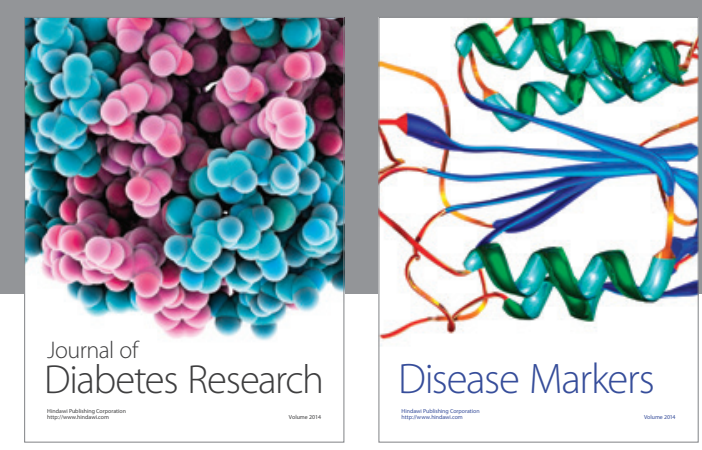

Disease Markers
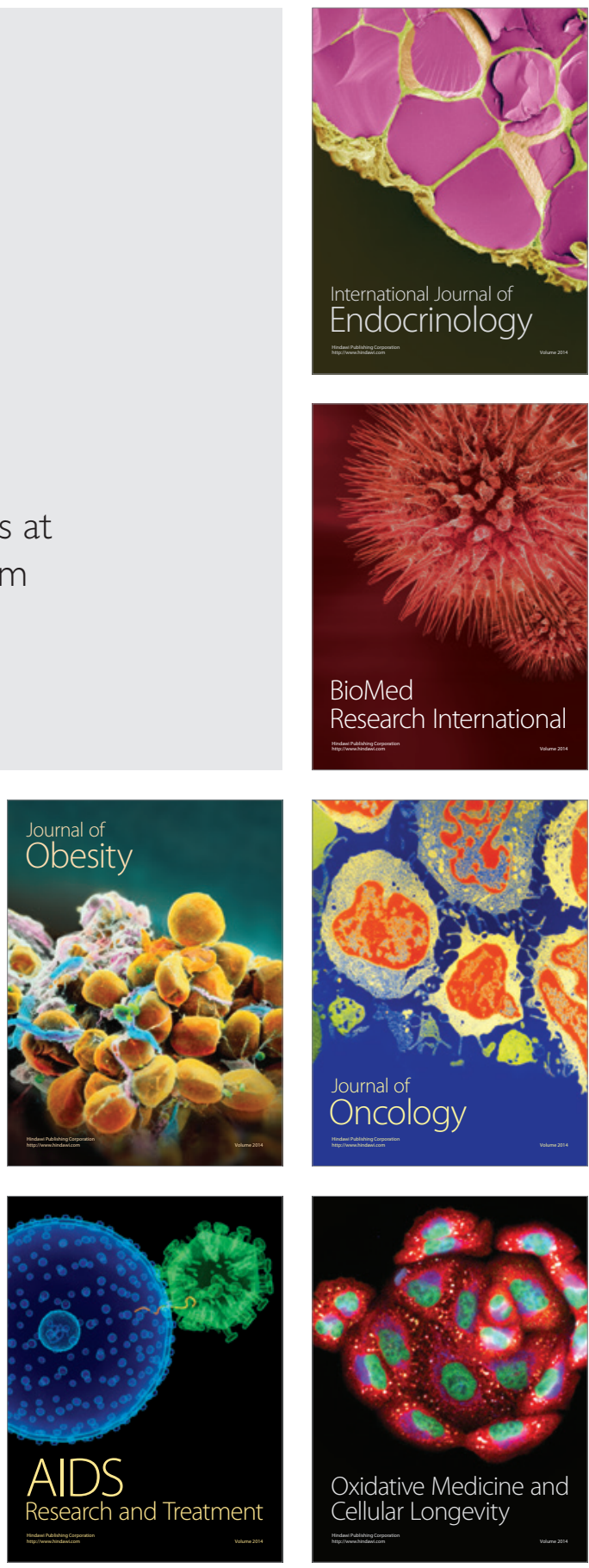